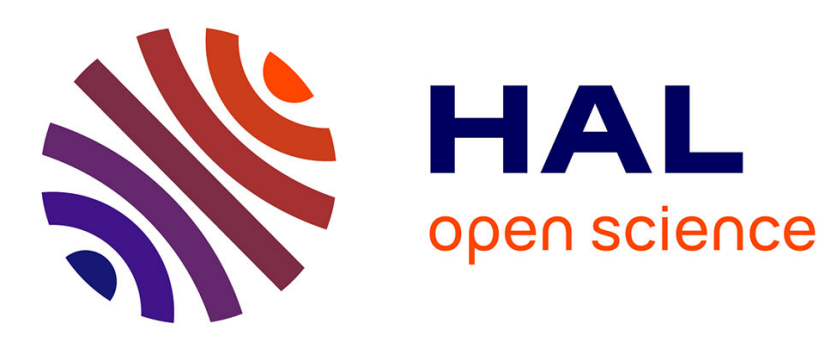

\title{
Etude en pression de la friction mutuelle dans l'hélium II
} P. Mathieu, E. Boccara, Y. Simon

\section{To cite this version:}

P. Mathieu, E. Boccara, Y. Simon. Etude en pression de la friction mutuelle dans l'hélium II. Journal de Physique Lettres, 1982, 43 (22), pp.785-791. 10.1051/jphyslet:019820043022078500 . jpa00232126

\section{HAL Id: jpa-00232126 https://hal.science/jpa-00232126}

Submitted on 1 Jan 1982

HAL is a multi-disciplinary open access archive for the deposit and dissemination of scientific research documents, whether they are published or not. The documents may come from teaching and research institutions in France or abroad, or from public or private research centers.
L'archive ouverte pluridisciplinaire HAL, est destinée au dépôt et à la diffusion de documents scientifiques de niveau recherche, publiés ou non, émanant des établissements d'enseignement et de recherche français ou étrangers, des laboratoires publics ou privés. 
Classification

Physics Abstracts

$67.40 \mathrm{~V}$

\title{
Etude en pression de la friction mutuelle dans l'hélium II
}

\author{
P. Mathieu, E. Boccara et Y. Simon \\ Groupe de Physique des Solides de l'Ecole Normale Supérieure (*), \\ 24, rue Lhomond, 75231 Paris Cedex 05, France
}

(Reçu le 28 juillet 1982, accepté le 28 septembre 1982)

\begin{abstract}
Résumé - Nous avons mesuré le coefficient de friction mutuelle $B$ en fonction de la pression entre $1,4 \mathrm{~K}$ et la ligne $\lambda$. Nos résultats sont confrontés avec deux théories hydrodynamiques de la friction mutuelle récemment publiées par les auteurs d'une part [2], et par Pitaevskii [6] et Sonin [7], pour la région critique, d'autre part.
\end{abstract}

\begin{abstract}
We report on the first measurements of the mutual friction parameter $B$ as a function of pressure, from $T=1.4 \mathrm{~K}$ up to the lambda line. Experimental results are compared with predictions of our hydrodynamic theory of mutual friction [2], and, for the critical region, with those of the Pitaevskii-Sonin theory $[6,7]$.
\end{abstract}

1. Introduction - Dans l'hélium II en rotation, une onde de deuxième son se propageant normalement à l'axe de rotation subit très nettement une atténuation supplémentaire. Depuis le travail fondamental de Hall et Vinen [1], on attribue cet effet à l'apparition d'un réseau uniforme de lignes de tourbillons superfluides (ou vortex) parallèles à l'axe de rotation. Le coefficient d'atténuation du $2^{\mathrm{e}}$ son, qu'on peut écrire $\alpha=\alpha_{0}+B \Omega / 2 u_{2}$, varie proportionnellement à la vitesse angulaire $\Omega$, donc à la densité de vortex $2 \Omega / K ; u_{2}$ est la vitesse du $2^{\mathrm{e}}$ son, $K$ le quantum de circulation, et $B$ un coefficient sans dimension. Un vortex plongé dans le champ de vitesses normal-superfluide $\mathbf{v}_{n_{1}}$ et $\mathbf{v}_{s_{1}}$ d'une onde de $2^{e}$ son oscille avec une vitesse $v_{L}$ qui n'est ni $v_{n_{1}}$, ni $v_{s_{1}}$ [2] ; ces petits mouvements sont dissipatifs. A grande échelle tout se passe comme s'il y avait, en présence des vortex, une friction mutuelle entre les deux écoulements incidents. Pour étudier la dynamique d'un vortex, c'est-à-dire pour exprimer $v_{L}$ en fonction de $v_{n_{1}}$ et $v_{s_{1}}$, Hall et Vinen ont introduit deux coefficients cinétiques. Le premier est justement $B$. Le second, $B^{\prime}$, n'intervient pas dans la dissipation et ses effets, analogues à ceux de la force de Coriolis, n'apparaissent que dans des circonstances expérimentales exceptionnelles [3].

Les mesures connues de $B$ et $B^{\prime}$ en fonction de la température, de $T=1,4 \mathrm{~K}$ à quelques dixièmes de $\mathrm{mK}$ de $T_{\lambda}$ [3-5], ont toutes été faites sous pression de vapeur saturante (PVS). Nous donnons ici les premières mesures de $B$ en fonction de la pression de 0 à 24 bars, entre $T=1,4 \mathrm{~K}$ et la ligne $\lambda$; leur intérêt est avant tout de tester les théories hydrodynamiques récemment publiées, par les auteurs d'une part [2], et par Pitaevskii et Sonin d'autre part [6, 7]. Une brève revue de la théorie est nécessaire pour la discussion.

(*) Laboratoire associé au Centre National de la Recherche Scientifique. 
2. Les théories de la friction mutuelle. - La première théorie qui fut proposée en 1956 par Hall et Vinen eux-mêmes [1] est essentiellement une théorie cinétique : on considère que l'interaction du fluide normal avec le superfluide via le vortex résulte des collisions entre les rotons (supposés sans interaction) et le cœur du vortex. $B$ et $B^{\prime}$ sont exprimés en fonction de deux sections efficaces de collision $\sigma_{\|}$et $\sigma_{\perp}$. Après Hall et Vinen, plusieurs théoriciens ont cherché à calculer les $\sigma$ [8], mais à l'évidence aucune des valeurs théoriques proposées ne rend bien compte des valeurs expérimentales de $B(T)$ et $B^{\prime}(T)$ (hormis leur ordre de grandeur qui est l'unité; voir Fig. 1 dans [8]). En fait un traitement cinétique des collisions rotons-vortex suppose que le libre parcours moyen $L$ des rotons reste grand devant le rayon $a$ du cœur du vortex $(a \sim 10 \AA)$; la condition $L \gg a$ limite donc strictement aux basses températures $(T \lesssim 1,6 \mathrm{~K})$ la validité d'une théorie cinétique. D'autre part, pour achever le calcul de $B$ et $B^{\prime}$ en fonction de $\sigma_{\perp}$ et $\sigma_{\|}$, Hall et Vinen doivent faire appel à des notions et résultats d'hydrodynamique classique, en supposant que le vortex se comporte un peu comme une corde vibrante dans un fluide visqueux. Cette analogie, bien que physiquement fondée, apparaît dans un modèle cinétique comme une grossière approximation. Malgré ses imperfections et ses limites, le modèle de Hall et Vinen, avec quelques variantes [8], est resté jusqu'à une date récente la seule théorie de référence en la matière, et fait autorité encore aujourd'hui.

Une autre démarche, purement hydrodynamique, est celle que nous avons adoptée. Elle consiste à s'appuyer uniquement sur les équations du mouvement du modèle à deux fluides (Eqs. de Landau-Khalatnikov) et à chercher une solution de ces équations capable de décrire un vortex oscillant dans un champ $\mathbf{v}_{n_{1}}, v_{s_{1}}$. Nous trouvons que $B^{\prime}=0$ et que $B$ s'exprime en fonction de paramètres macroscopiques connus, tels que la viscosité du fluide normal $\eta$ et les « densités " normale et superfluide $\rho_{\mathrm{n}}$ et $\rho_{\mathrm{s}}\left(\rho_{\mathrm{n}}+\rho_{\mathrm{s}}=\rho\right)$ :

$$
B=\frac{16 \pi \eta \rho}{\rho_{\mathrm{n}} \rho_{\mathrm{s}} K} 1 / \log \left[\eta / \rho_{\mathrm{n}} \omega l^{2}\right],
$$

où $\omega / 2 \pi$ est la fréquence du son, et $l$ un rayon de coupure [2], dont la valeur exacte dépend de la structure du cœur mais qui reste voisine de $1 \AA$. A la précision usuelle des mesures de $B(\simeq 5 \%)$ $l$ intervenant sous un logarithme, n'est pas véritablement un paramètre ajustable.

La formule (1) est en très bon accord avec les valeurs expérimentales de $B(T)$ entre 1,7 et $2,1 \mathrm{~K}$ (pour $\omega \simeq 10^{4} \mathrm{~s}^{-1}$ ) et $B^{\prime}$ ne s'écarte effectivement de zéro de façon significative qu'en dehors de cet intervalle. Les limites de validité en température s'expliquent aisément. Vers les basses températures, le libre parcours moyen des rotons augmente, et, à l'inverse de la théorie cinétique, une théorie hydrodynamique devient inadéquate quand $L \gtrsim a$, rayon du coeur. D'un autre côté, lorsque $T \rightarrow T_{\lambda}$, il faut tenir compte dans les équations hydrodynamiques des effets de relaxation de la densité superfluide [11].

En supposant que le mécanisme dissipatif dominant dans la région critique est précisément la relaxation de $\rho_{\mathrm{s}}$ avec un temps caractéristique $\tau$, Pitaevskii [6] montre que :

$$
B^{-1} \sim \tau \rho_{\mathrm{s}}\left(\partial \mu_{\mathrm{s}} / \partial \rho_{\mathrm{s}}\right)
$$

où $\mu_{\mathrm{s}}$ est le potentiel chimique associé à $\rho_{\mathrm{s}}: \tau, \rho_{\mathrm{s}}$ et $\partial \mu_{\mathrm{s}} / \partial \rho_{\mathrm{s}}$ ayant des exposants critiques universels respectivement voisins de $-1,2 / 3$ et $2 / 3$, il en résulte que $B$ doit diverger, lorsque

$$
\varepsilon=\left(T_{\lambda}-T\right) / T_{\lambda} \rightarrow 0
$$

avec un exposant critique - 1/3, en accord avec les mesures sous PVS [3]. Le calcul hydrodynamique complet de $B$ et $B^{\prime}$, par Sonin, conduit à des expressions $B=b \varepsilon^{-1 / 3}$ et $B^{\prime}=b^{\prime} \varepsilon^{-1 / 3}$, avec toujours l'exposant - $1 / 3$; mais les valeurs théoriques de $b$ et $b^{\prime}(0,63$ et $-0,93)$ s'écartent assez nettement des valeurs expérimentales [3] $\left(b=0,47\right.$ et $\left.b^{\prime}=-0,34\right)$. Notons que (1) extrapolée dans la région critique donne des valeurs trop élevées de $B$ avec un exposant critique $-2 / 3$. 
En étendant les mesures de $B$ dans le domaine de phase (pression et température) de l'hélium II, nous avions un double objectif :

1) obtenir un test quantitatif supplémentaire de notre modèle hydrodynamique. En effet, d'après (1) et compte tenu des variations de $\eta(P)$ et $\rho_{\mathrm{n}}(P)$, on prévoit entre 1,8 et $2,1 \mathrm{~K}$, une forte augmentation de $B$ avec la pression $P$.

2) vérifier, le long de la ligne $\lambda$, l'existence d'un exposant critique universel $-1 / 3$, indépendant de $P$.

3. Principe expérimental. - La technique de mesure de $B$ est classique et nous l'avons discutée en détail ailleurs $[3,9,12]$. Une cavité parallélépipédique $\left(6 \times 23 \times 58 \mathrm{~mm}^{3}\right)$ est excitée sur le mode fondamental $\omega_{0}=\pi u_{2} / d$ correspondant à une de ses dimensions horizontales $(d=6$ ou $58 \mathrm{~mm})$. On compare les facteurs de qualité de la résonance, à l'arrêt et en rotation, soit $Q_{0}=\pi / \alpha_{0} d$ et $Q=\pi / \alpha d$. On en déduit $B=2 \omega \Omega^{-1}\left|1 / Q-1 / Q_{0}\right|$.

Comme $u_{2}$ est fonction de $P$ et $T$, le maintien sur une résonance interdit toute dérive de température ou de pression. Ainsi à $1,9 \mathrm{~K}$, avec un $Q_{0} \simeq 10^{4}$ typiquement, une fluctuation $\delta T \simeq 10^{-5} \mathrm{~K}$, ou $\delta P \simeq 1$ mbar, ferait varier la fréquence de résonance $\omega_{0}$ d'une quantité comparable à la largeur de résonance $\omega_{0} / Q$. D'habitude, on travaille sous PVS et il suffit de contrôler $T$. La difficulté consistait ici à construire un montage tournant $(\Omega \simeq 1-10 \mathrm{rad}$./s) où on puisse stabiliser rigoureusement la pression à une valeur quelconque entre 0 et 25 bars.

Le vase tournant qui entraîne l'échantillon d'He II sous pression $\left(750 \mathrm{~cm}^{3}\right)$ avec le résonateur est un cylindre de cuivre étanche, qu'on peut relier à l'arrêt à une bouteille d'hélium comprimé. La pression finale après remplissage, mesurée grâce à une jauge capacitive Datametrics 1018, est déterminée à \pm 30 mbar. Avant la mise en rotation, le cylindre est fermé par une vanne aiguille étanche au superfluide, manipulée de l'extérieur par une commande coaxiale avec l'arbre de rotation et le capillaire de remplissage. On enferme ainsi une masse homogène d'hélium entièrement immergée dans le bain principal dont la température est régulée comme d'habitude sous PVS. La vanne aiguille est certainement la pièce essentielle et originale du montage. L'expérience montre que si on se contente d'isoler le cylindre par une vanne extérieure, les variations incontrôlables de température le long du capillaire, de même qu'une microfuite quelconque, suffisent à provoquer une dérive de pression immédiatement détectable par le résonateur.

Dans la région critique, et partout où $u_{2}$ varie rapidement avec $T$, nous utilisons un système de contrôle de la température mis au point au laboratoire et qui a l'avantage sur les régulations classiques à pont d'éviter toute dérive lente de température. Il consiste à asservir $T$ sur la résonance aiguë d'une cavité $2^{\mathrm{e}}$ son auxiliaire $(Q \simeq 30000)$ placée dans le bain principal : l'écart à la résonance fournit le signal d'erreur qui alimente, après amplification, une résistance chauffante.

Si on veut s'approcher de la ligne $\lambda$, par exemple à $1,9 \mathrm{~K}$, on porte la pression dans le cylindre à une valeur un peu inférieure à 21,4 bars, pression pour laquelle $T_{\lambda}(P)=1,9 \mathrm{~K}$ (Fig. 2). La vanne est fermée, et on commande la température en diminuant légèrement, sur le clavier d'un synthétiseur, la fréquence d'excitation de la cavité de régulation. A $1,9 \mathrm{~K}$, la stabilité en température (dans la bande $0-1 \mathrm{~Hz}$ ) est meilleure que $10^{-8} \mathrm{~K}$. Cette méthode permet donc de fixer puis de varier la température avec une grande sensibilité mais, pas plus que la mesure directe de $T$ par des moyens classiques (mesure de PVS), elle ne permet pas d'apprécier la valeur absolue de l'écart $T_{\lambda}-T \lesssim 10^{-2} \mathrm{~K}$. Il se trouve que la vitesse du $2^{\mathrm{e}}$ son directement déduite de $\omega_{0}\left(u_{2}=\omega_{0} d / \pi\right)$ constitue un excellent thermomètre dans la région critique. En effet, $u_{2}$ décroît rapidement vers zéro au point $\lambda$ suivant une loi empirique bien établie [13] :

$$
u_{2}(P, \varepsilon)=f(P) \varepsilon^{1 / 3}\left[1+g(P) \varepsilon^{1 / 6}\right], \quad\left(\varepsilon<10^{-2}\right),
$$

où $f$ et $g$ sont deux polynômes en $P$. Connaissant $u_{2}$ et $P$, on calcule $\varepsilon$. Il est important de noter que lorsqu'on exprime une grandeur telle que $u_{2}$ ou $B$ en fonction de $P$ et $T$, seule la variable $\varepsilon$ 
est critique; c'est-à-dire qu'à $\varepsilon$ constant, $u_{2}(f$ et $g)$ et $B$ sont des fonctions de $P$ à variation lente. L'incertitude sur la pression initiale ( \pm 30 mbar) n'entraîne pas d'erreur appréciable sur $\varepsilon$. On peut également ignorer le $\Delta P$, au maximum quelques dizaines de millibars, qui accompagne l'augmentation de température après la fermeture de la vanne (on suit une isochore sur $10^{-2} \mathrm{~K}$ ).

4. Résultats et discussion. -4.1 LoIN $D E T_{\lambda}$ - $-B$ a été mesuré jusqu'à $P=24$ bars, à partir de $1,4 \mathrm{~K}$ jusqu'à $50 \mathrm{mK}$ environ de la ligne $\lambda$, et pour une fréquence moyenne du son de $1 \mathrm{kHz}$ qui correspond à la petite dimension du résonateur $(d=6 \mathrm{~mm})$. Les résultats, regroupés dans le tableau I, ont été comparés aux $B$ calculés par la formule (1). Alors que $\rho_{n}, \rho_{\mathrm{s}}$ et $\rho$ sont très bien connues à toute pression, les seules mesures de $\eta(P)$ dont nous disposons sont celles de Brewer et Edwards [14], qui sont incomplètes au-dessus de $1,8 \mathrm{~K}$ et relativement imprécises. Malgré ces incertitudes sur $\eta$, l'équation (1) est remarquablement vérifiée au-dessus d'une température limite variable avec $P$ de 1,7 à 1,5 K. La figure 1 montre, par exemple, les points expérimentaux à $T=1,8 \mathrm{~K}$ avec la courbe théorique $B(P)$; dans (1), nous avons pris $l=0,8 \AA$ [9] et à chaque pression la valeur réelle de $\omega$ et non une valeur moyenne. En vérifiant (1), nous confirmons une des conclusions les plus importantes de notre modèle : l'écoulement du fluide normal est perturbé au voisinage du cœur du vortex sur une distance de l'ordre de $\delta=\left(\eta / \rho_{n} \omega\right)^{1 / 2}$ (profondeur de peau visqueuse) et le mécanisme dissipatif de la friction mutuelle n'est rien d'autre que la viscosité du fluide normal dans cette région perturbée.

Tableau I.

\begin{tabular}{|c|c|c|c|c|c|c|c|c|c|}
\hline $\begin{array}{r}\text { P(bars) } \\
\text { T(K) }\end{array}$ & PVS & 3 & 6 & 9 & 12 & 15 & 18 & 21 & 24 \\
\hline 1,4 & 1,50 & 1,51 & 1,53 & 1,52 & 153 & 1,53 & 1,51 & 1,48 & 1,47 \\
\hline 1,5 & 1,34 & 1,36 & 1,34 & 1,35 & 1,34 & 1,32 & 1,29 & 1,29 & 1,28 \\
\hline 1,6 & 1,20 & 1,22 & 1,20 & 1,22 & 1,24 & 1,25 & 1,26 & 1,25 & 1,27 \\
\hline 1,7 & 1,07 & 1,08 & 1,09 & 1,12 & 1,12 & 1,16 & 1,20 & 1,28 & 1,43 \\
\hline 1,8 & 0,96 & 0,99 & 1,04 & 111 & 1,16 & 1,23 & 1,39 & 1,70 & 2,17 \\
\hline 1,9 & 0,94 & 1,03 & 1,13 & 1,25 & 1,40 & 1,72 & 2,22 & & \\
\hline 2,0 & 1,03 & 1,19 & 1,40 & 1,68 & 2,26 & & & & \\
\hline 2,1 & 1,65 & 1,95 & & & & & & & \\
\hline
\end{tabular}

Sur la figure 2, nous avons reporté dans le plan $(P, T)$ les points de mesure du tableau I en notant par une croix tous ceux pour lesquels l'accord entre théorie et expérience est voisin ou meilleur que $10 \%$ A $1,7 \mathrm{~K}$, sous PVS, on est à la limite du domaine de validité. A $1,5 \mathrm{~K}$, toujours sous PVS, il y a déjà presque un facteur 2 entre le $B$ théorique et le $B$ expérimental; mais à 25 bars, on retrouve la valeur théorique à mieux que $10 \%$. Ceci s'explique assez bien, comme nous allons le voir, par une diminution du libre parcours moyen des rotons $L$ avec la pression.

On peut estimer $L$ par la formule cinétique classique qui donne la viscosité :

$$
L=\frac{3 \eta}{\rho_{\mathrm{n}} v_{\mathrm{r}}} \quad\left(v_{\mathrm{r}}=[2 k T / \pi \mu]^{1 / 2}\right)
$$

où $v_{\mathrm{r}}$ est la vitesse thermique des rotons, et $\mu$ leur masse effective. $L$ doit être comparé au rayon du coeur $a$, c'est-à-dire la distance typique sur laquelle la densité superfluide $\rho_{\mathrm{s}}^{\prime}(r)$ passe de zéro au centre du vortex à sa valeur dans la masse $\rho_{\mathrm{s}}$. Un calcul théorique permet d'apprécier le profil 


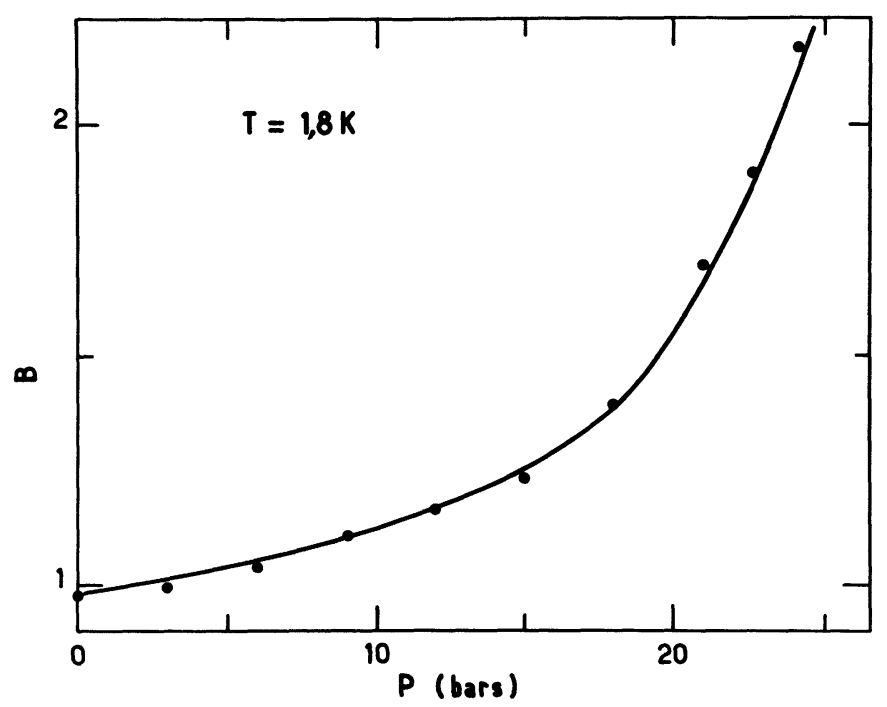

Fig. 1. - Les points représentent les mesures de $B$ en fonction de la pression à $T=1,8 \mathrm{~K}$. La courbe représente la fonction $B(P)$ théorique d'après l'équation (1).

[Experimental points : mutual-friction parameter $B$ as a function of the pressure at $T=1.8 \mathrm{~K}$. The solid line is the theoretical curve derived from the hydrodynamic formula (1).]

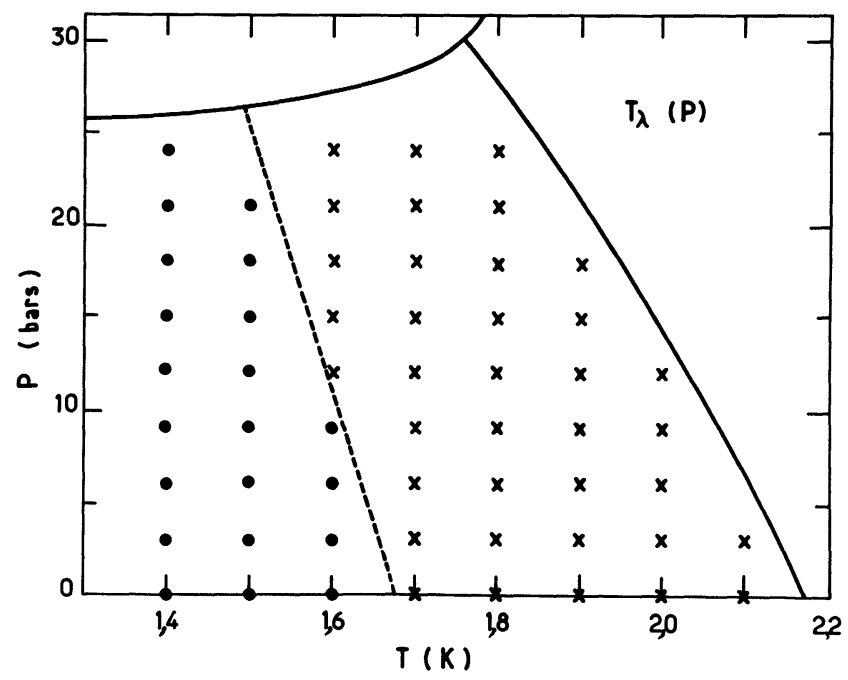

Fig. 2. - On a reporté dans le plan $(P-T)$ les points de mesure du tableau I en notant une croix chaque fois que l'accord entre la mesure et la valeur théorique (1) de $B$ est voisin ou meilleur que $10 \%$ (et un point dans le cas contraire). La ligne en pointillés est une ligne $L=11 \AA=C$ te, calculée à partir de (3).

[The measured points of table I are plotted in the $(P-T)$ plane as crosses when the theoretical value of $B$ from equation (1) agrees with experiment to better than $10 \%$ and as dots for the other results. The dashed line represents the line $L=11 \AA=$ constant such as calculated from equation (3).]

$\rho_{\mathrm{s}}^{\prime}(r)$ [15], et si on définit $a$ par $\rho_{\mathrm{s}}^{\prime}(a)=0,9 \rho_{\mathrm{s}}$, on trouve que $a$ varie peu autour de $10 \AA$ dans la gamme de températures qui nous intéresse ici. Cet ordre de grandeur est d'ailleurs confirmé 
expérimentalement [12]. Ainsi de 1,7 à $1,4 \mathrm{~K}$ sous PVS, le calcul montre que $a$ décroît très peu de 8 à $6 \AA$, tandis que $L$ d'après (3) augmente d'un facteur 4 (de 9 à $36 \AA$ ). A $1,5 \mathrm{~K}$, de $P=0$ à 25 bars, $L$ est divisé par 2 (de 22 à $11 \AA$ ). Une ligne $L=11 \AA=$ Cte (en pointillés sur la figure 2) marque à peu près la frontière du domaine hydrodynamique.

Sans prétendre à la rigueur, ces chiffres suggèrent que la condition $L \lesssim a$ est le bon critère d'application de l'hydrodynamique à la dynamique du vortex. Tout au moins, qualitativement, ils montrent que la décroissance de $L / a$ avec $T$ est bien la raison qui limite aux basses températures la validité de notre modèle.

4.2 LA RÉGION CRITIQUE. - Nous avons étudié le comportement critique de $B$ pour des valeurs de $\varepsilon$ comprises entre $10^{-2}$ et $10^{-4}$. La fréquence moyenne du $2^{\mathrm{e}}$ son, $30 \mathrm{~Hz}$, correspond à la grande longueur du résonateur $(d=58 \mathrm{~mm})$. Ainsi, nous nous plaçons à peu près dans la même gamme de fréquences que dans nos précédentes mesures sous PVS [3]. Comme sous PVS, nous trouvons que $B$ obéit à une loi de la forme (exemple Fig. 3) :

$$
B=b(P) \varepsilon^{-x(P)} \quad\left(10^{-4} \lesssim \varepsilon \lesssim 10^{-2}\right),
$$

où $b(P)$ et $x(P)$ sont des fonctions lentement variables de la pression. Le tableau II donne les valeurs de $b$ et $x$ sous PVS [3] et pour 3 valeurs de la pression.

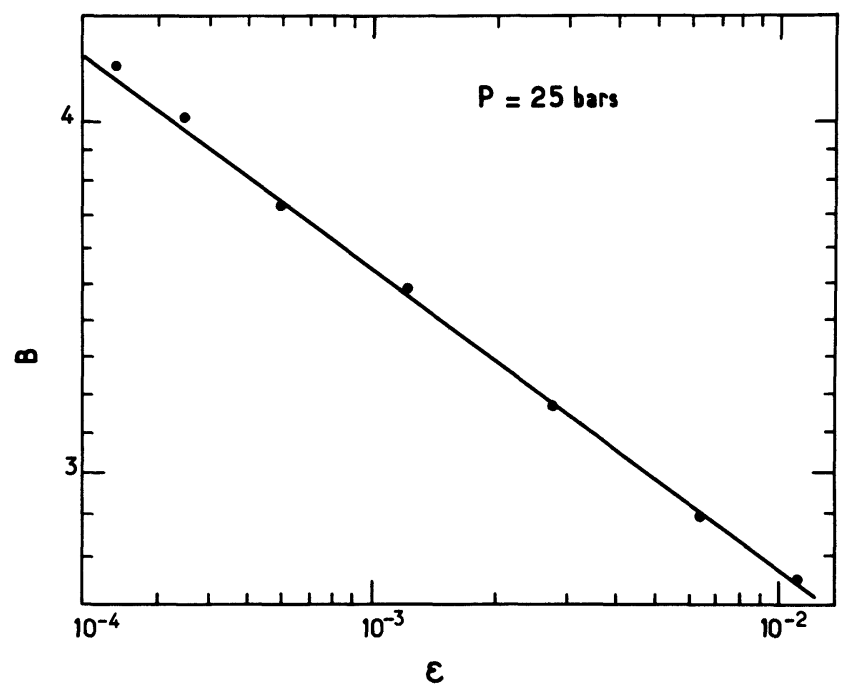

Fig. 3. - Coefficient de friction mutuelle $B$ en fonction de la température réduite $\varepsilon=\left(T_{\lambda}-T\right) / T_{\lambda}$ à $P=25$ bars (échelles logarithmiques). La droite correspond à une loi de la forme (4) avec $b=1,52$ et $x=0,11$.

[Mutual-friction parameter $B$ as a function of the reduced temperature $\varepsilon=\left(T_{\lambda}-T\right) / T_{\lambda}$ on a logarithmic scale, at $P=25$ bars. The fitted line corresponds to a power-law dependence $B=b \varepsilon^{-x}$, with $b=1.52$ and $x=0.11$.

Tableau II.

\begin{tabular}{|c|c|c|l|l|}
\hline$P($ bars $)$ & PVS [3] & 11,5 & 15,5 & 25 \\
\hline$b( \pm 0,03)$ & 0,47 & 0,84 & 1,23 & 1,52 \\
\hline$x( \pm 0,02)$ & 0,33 & 0,22 & 0,17 & 0,11 \\
\hline
\end{tabular}


Le résultat le plus marquant est la variation inattendue de l'exposant critique $x$ avec la pression, en contradiction avec les conclusions de Pitaevskii [6] et Sonin [7].

On peut se demander si, à $\varepsilon \simeq 10^{-4}$, on n'est pas encore trop loin du point $\lambda$ pour mettre en évidence l'exposant critique dominant. Notons cependant que le « bon » exposant - 0,33 que nous avions trouvé avec Serra [3], et qui est le seul résultat expérimental sur lequel s'appuient Pitaevskii et Sonin, a été obtenu dans la même gamme de $\varepsilon$ et avec les mêmes techniques. En pratique, on peut difficilement diminuer $\varepsilon$ en dessous de $10^{-4}$. En effet, pour rester dans le domaine de l'acoustique linéaire, on doit baisser l'amplitude du $2^{e}$ son et le signal détecté est trop faible. D'autre part, la variation de pression gravitationnelle entraîne une inhomogénéité de l'hélium dans la cavité $\left(\mathrm{d} \varepsilon / \mathrm{d} h \simeq 10^{-6} / \mathrm{cm}\right)$. Cette inhomogénéité devient sensible à partir de $\varepsilon \simeq 10^{-4}$ puisqu'elle se traduit par des variations de plus de $1 \%$ de toutes les grandeurs critiques, en particulier $u_{2}$.

Avant de présenter nos résultats comme une exception aux règles d'universalité des exposants critiques, il convient de mettre en cause les approximations faites par Sonin [7]. A $50 \mathrm{mK}$ de la ligne $\lambda\left(\varepsilon \simeq 2,5 \times 10^{-2}\right)$ l'expression hydrodynamique (1) rend encore très bien compte de la valeur expérimentale de $B$ (par exemple sous PVS à 2,12 K [2]). Encore une fois, ceci signifie qu'à $\varepsilon=2,5 \times 10^{-2}$ le seul mécanisme dissipatif en jeu dans la friction mutuelle est la viscosité du fluide normal. Si on extrapole (1) à $\varepsilon \simeq 10^{-4}$, on trouve une dissipation plus forte (un $B$ plus élevé) que celle qui est observée. Or Sonin néglige complètement la perturbation de l'écoulement normal (en posant rot $v_{n} \equiv 0$ ) et l'équation de Navier-Stokes disparaît de son système d'équations. L'approximation est sans doute un peu brutale. Dans la région $\varepsilon \simeq 10^{-4}-10^{-2}$, le problème devrait plutôt se poser dans les termes suivants : comment les effets de relaxation, tout en apportant leur mécanisme dissipatif propre, peuvent-ils modifier la dynamique du vortex et notamment la forme de l'écoulement du fluide normal autour du cœur dans un sens qui réduit la dissipation visqueuse ? Ce problème résolu, on devrait retrouver certains résultats constructifs de la théorie de Sonin en particulier l'existence d'un $B^{\prime}$ négatif du bon ordre de grandeur.

\section{Bibliographie}

[1] Hall, H. E. and Vinen, W. F., Proc. R. Soc. London A 238 (1956) 204 ; 238 (1956) 215.

[2] Mathieu, P. and Simon, Y., Phys. Rev. Lett. 45 (1980) 1428.

[3] Mathieu, P., Serra, A. and Simon, Y., Phys. Rev. B 14 (1976) 3753.

[4] LuCAs, P., J. Phys. C 3 (1970) 1180.

[5] Miller, R. J., Lynall, I. H. and Mehl, J. B., Phys. Rev. B 17 (1978) 1035.

[6] PitaevskiI, L. P., Pis'ma Zh. Eksp. Teor. Fiz. 25 (1977) 168.

[7] Sonin, E. B., J. Low Temp. Phys. 42 (1981) 417.

[8] Hiller, A. J., Hall, H. E. and Lucas, P., J. Phys. C 7 (1974) 3341.

[9] Mathieu, P. and Simon, Y., Phys. Rev. B 26 (1982) 1233.

[10] Vinen, W. F. and Donnelly, R. J., revue en préparation pour Progress in Low Temp. Phys.

[11] Ginzburg, V. L. and Sobyanin, A. A., Usp. Fiz. Nauk. 120 (1976) 153 ; Sov. Phys. Usp. 19 (1976) 773.

[12] Mathieu, P., Maréchal, J. C. and Simon, Y., Phys. Rev. B 22 (1980) 4293.

[13] Greywall, D. S. and Ahlers, G., Phys. Rev. A 7 (1973) 2145.

[14] Brewer, D. F. and Edwards, D. O., Proc. 8th Int. Conf. Low Temp. Phys., Editor Davies (Butterworths) 1963 , p. 96.

[15] D'Humières, D., Thèse, Paris (novembre 1980). 